Chinese Journal of Organic Chemistry

\title{
小分子蓝色磷光主体材料
}

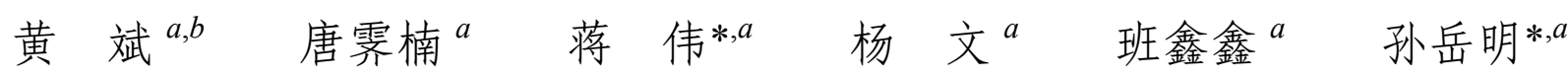 \\ $\left({ }^{a}\right.$ 东南大学化学化工学院 南京 211189) \\ ( ${ }^{b}$ 东南大学成贤学院化工与制药工程系 南京 210088)
}

\begin{abstract}
摘要 有机电致发光器件是有机光电子领域的研究热点, 在平板显示和固体照明领域有着广阔的应用前景. 目前, 由 于器件效率和稳定性的问题, 蓝色磷光器件是有机电致发光器件的瓶颈, 而蓝色磷光主体材料的选择是影响蓝色磷光 器件性能的关键因素. 综述了小分子蓝色磷光主体材料的最新研究进展, 重点介绍了各类小分子蓝色磷光主体材料的 设计思想、器件性能. 包括空穴传输性主体材料、含硅主体材料、电子传输性主体材料、双极主体材料和可湿法加工 的蓝色磷光小分子主体材料的结构、特点及相应器件性能. 最后对小分子蓝色磷光主体材料的发展方向进行了展望. 关键词＼cjkstart有机电致发光; 蓝色磷光; 有机小分子; 主体材料; 空穴; 电子; 双极; 湿法
\end{abstract}

\section{Organic Small Molecules Host Materials for Blue Phosphorescent Organic Light-Emitting Diodes}

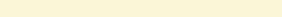 \\ Huang, $\operatorname{Bin}^{a, b} \quad$ Tang, Jinan ${ }^{a} \quad$ Jiang, Wei ${ }^{*, a} \quad$ Yang, Wen ${ }^{a}$ \\ Ban, Xinxin $^{a}$ Sun, Yueming ${ }^{*, a}$ \\ ( ${ }^{a}$ School of Chemistry and Chemical Engineering, Southeast University, Nanjing 211189) \\ ( ${ }^{b}$ Department of Chemical and Pharmaceutical Engineering, Southeast University Chengxian College, Nanjing 210088)
}

\begin{abstract}
Organic light emitting diodes (OLEDs) have gained tremendous attention due to their significant potential applications in flat-panel displays and solid state lighting. Blue electrophosphorescent devices are bottlenecks of research of OLEDs because of efficiency and stability, the choice of host materials for blue electrophosphorescence is very important. This review summarizes the recent progress in organic small molecules host materials for blue phosphorescent organic light-emitting diodes, focusing on the design strategies and device performance. Different type host materials including hole-transport-type host materials, silane-based host materials, electron-transport-type host materials, bipolar transport host materials and solution-processed host materials for blue electrophosphorescence are summarized with their structures, features and corresponding devices performance. Finally, the direction of development of organic small molecule host materials for blue electrophosphorescence is prospected.
\end{abstract}

Keywords organic light emitting diodes; blue electrophosphorescence; organic small molecules; host materials; hole; electron; bipolar; solution-processed

有机电致发光器件(OLED) 是有机光电子领域的研 究热点, 由于其自发射性能强、响应时间短、视角广、 高对比度、质量轻等优点, 在下一代平板显示和固体照 明技术中, 具有广阔的应用前景 ${ }^{[1]} .1998$ 年, 我国吉林 大学的马於光教授 ${ }^{[2]}$ 和美国普林斯顿大学的 Forrest 教 授 ${ }^{[3]}$ 分别报道了采用锇配合物和铂配合物作为染料掺杂 入发光层, 第一次成功得到并解释了磷光电致发光现
象. 他们分别利用锇和铂的重原子效应以提高分子自 旋-轨道耦合，从而充分利用器件中的单线态和三线态 激子, 突破了传统有机电致发光理论内量子效率低于 $25 \%$ 的限制, 使器件的理论最大内量子效率达到 $100 \%$, 从而开辟了电致磷光这一新的研究领域.

目前, 有机小分子电致磷光器件中, 绿色磷光和红 色磷光器件取得了较大的进展, 发现了许多可用于有机

\footnotetext{
*E-mail:101011462@seu.edu.cn

Received December 9, 2012; revised January 29, 2013; published online February 5, 2013

Project supported by the National Natural Science Foundation of China (Nos. 51103023, 21173042), the National Basic Research Program of China (973 Program, No. 2013CB932900).

国家自然科学基金(Nos. 51103023, 21173042)、国家重点基础研究发展计划(973 计划, No. 2013CB932900)资助项目.
} 
电致磷光二极管(PhOLEDs)的绿光和红光材料, 用这些 材料制成的 PhOLEDs 具有发光效率高、色度纯和使用 寿命长等优点. 作为固体照明和全彩显示器的重要组成 部分, 蓝光 PhOLEDs 方面的进展不如绿光和红光 PhOLEDs $^{[4]}$. 高效的、稳定的蓝光发光器件是限制 PhOLEDs 大规模应用的最大障碍 ${ }^{[5]}$.

小分子蓝光 PhOLEDs 性能还有待进一步提高, 制 约因素之一是性能优良的小分子主体材料的选择 ${ }^{[6]}$. 首 先, 适合于蓝色磷光的主体材料的三线态能级必须比客 体的三线态能级高, 一般要求大于 $2.75 \mathrm{eV}$, 这样才能够 保证主体材料与蓝色磷光客体材料之间的能量传递为 放热过程, 客体没有能量回传给主体材料; 其次, 蓝色 磷光主体材料分子的最高占有轨道能级(HOMO) 和最低 未占轨道能级(LUMO) 要与器件中的相邻功能层相匹 配, 这样才有利于空穴和电子注入, 同时分子的单线态 能级与三线态能级要比较接近, 从而降低驱动电压; 再 次, 蓝色磷光主体材料分子应该具有良好的载流子传输 特性, 使电子和空穴在发光层中能有效地复合; 另外, 适合于蓝色磷光的主体材料应该具有良好的热、电化学 稳定性和优良的成膜性能.

近 10 年来, 基于小分子的蓝光 PhOLEDs 取得了较 大的进展, 已经有了这方面的综述 ${ }^{[59]}$. 蓝色磷光主体 材料是影响蓝光 PhOLEDs 性能的关键因素, 随着双极 小分子主体材料和可湿法成膜的小分子主体材料的迅 速发展, 蓝光 PhOLEDs 性能不断提高. 本文从主体材 料的设计与器件性能方面, 重点总结和评述了近 5 年小 分子蓝色磷光主体材料的特征及器件性能.

\section{1 小分子蓝色磷光主体材料研究进展}

\section{1 空穴传输性蓝色磷光主体材料}

\subsection{1 基于咔唑的空穴传输性蓝色磷光主体材料}

咔唑是蓝色磷光主体材料中常见的分子片段. 由于 咔唑基团具有较小的共轭体系, 其 $\mathrm{N}$ 原子上的孤对电子 能有效地降低分子的单线态和三线态能级分裂，咔唑基 团的三线态能级为 $3.02 \mathrm{eV}^{[10]}$.

最早报道的含咔唑基团的蓝色磷光主体材料是 $4,4^{\prime}-N, N^{\prime}$-二咔唑基联苯(CBP, 1$)^{[11]}$, 使用的磷光染料是 双 (4,6-二氟苯基吡啶- $N, \mathrm{C}^{2}$ ) 吡啶甲酰合铱(FIrpic). 由于 CBP 的三线态能级 $(2.56 \mathrm{eV})$ 低于 FIrpic 的三线态能级 $(2.62 \mathrm{eV}), \mathrm{CBP}$ 与 FIrpic 之间的能量传递为吸热能量传 递过程, FIrpic 可以将激发能量反向传递给主体材料, 使器件的效率受到不利影响. 器件的最高功率效率为 $6.3 \mathrm{~lm} / \mathrm{W}$, 外量子效率为 $5.7 \%$. 当使用 $N, N^{\prime}$ 二二咔唑基3,5-苯 $(\mathrm{mCP}, 2)$ 作为主体材料 ${ }^{[12]}$, 该主体材料的三线态 能级为 $2.90 \mathrm{eV}$, 主体材料与磷光染料之间的能量传递
为放热能量传递, 磷光染料不会将激发能量反向传递给 主体材料. 用 $\mathrm{mCP}$ 作为主体材料掺杂 FIrpic 制备的蓝光 器件 ${ }^{[13]}$, 亮度为 $100 \mathrm{~cd} / \mathrm{m}^{2}$ 时, 功率效率达到 $28.7 \mathrm{~lm} / \mathrm{W}$, 电流效率达到 $33.9 \mathrm{~cd} / \mathrm{A}$, 外量子效率为 $16.6 \%$.

为了提高 $\mathrm{CBP}$ 分子的三线态能级，在该分子的基 础上，通过引入亚甲基、醚键、环己基，改变基团的取 代位点,或者通过增加空间位阻将联苯的两个苯环错开, 破坏原来共平面的结构来减少共轭效应，制备了一系列 蓝色磷光主体材料 ${ }^{[14 ~ 16]}$, 如图 1 所示, 4,4'-二(9-咔唑 基)-2,2'-二甲基-联苯(CDBP, 3), 4,4'-二(9-咔唑基)-3,3'二甲基联苯 (o-CDBP, 4), 双 [4-(9H-咔唑-9-基)苯基]甲烷 (CBPM, 5), 二[4-(9-咔唑基)苯基]醚(CBPE，6)等. 经实 验测量, 该类分子的三线态能级都接近 $3.0 \mathrm{eV}$, 掺杂 FIrpic 的蓝光器件的电流效率最高达到 $20.4 \mathrm{~cd} / \mathrm{A}$, 外量 子效率提升至 $10.4 \%$. 值得注意的是, $o$-CDBP 分子的三 线态能级只有 $2.73 \mathrm{eV}$, 在该分子中引入的甲基并没有 起到破坏联苯共轭的目的, 而 $\mathrm{CBP}$ 的三线态能级主要 是由中间的联苯基团决定的.

台湾的 Tsai 等 ${ }^{[17,18]}$ 报道了对咔唑 3,6-位修饰的多个 衍生物, 如 3,6-二(9-咔唑基)-9-(2-乙基已基)咔唑(TCz1, 7), 9-苯基-3,6-二(三苯甲基)咔唑( $\mathrm{CzC}, 8$ )等, 分子式如 图 1 所示. 这类材料三线态能级在 $3.0 \mathrm{eV}$ 左右，应用于 蓝色磷光器件, 获得电流效率最高达到 $31 \mathrm{~cd} / \mathrm{A}$, 功率 效率达到 $28 \mathrm{~lm} / \mathrm{W}$, 外量子效率达到 $15 \%$. 从咔唑基团 的 9-位引入刚性共轭基团同样是制备蓝色磷光主体材 料的重要思路之一, $\mathrm{CBP}$ 和 $\mathrm{mCP}$ 及其衍生物就是很好的 例子. 在图 1 所示的结构中, $N$-苯基咔唑(CZPH, 9), 1,4二(9-咔唑基)甲基苯(DCB， 10)及 2,6,14-三(9-咔唑基)三 蝶烯(TCTP, 11)的三线态能级与咔唑分子保持一致, 为 $2.9 \mathrm{eV}$ 左右 ${ }^{[19,20]}$. $Z$-型寡聚苯衍生物 $2,2^{\prime}$-二(4-咔唑基苯 基)-1,1'-联苯(4CZPBP, 12)的三线态能级为 $2.8 \mathrm{eV}^{[21]}$, 应 用于蓝色磷光器件, 获得电流效率最高达到 $50.5 \mathrm{~cd} / \mathrm{A}$, 功率效率达到 $47.0 \mathrm{~lm} / \mathrm{W}$, 外量子效率达到 $22 \%$.

3,3'-二咔唑衍生物也可以用作空穴传输性蓝色磷光 主体材料. Sasabe 等 ${ }^{[13]}$ 在 3,3'-二咔唑分子的 9,9'-分别引 入苯基和四苯甲基, 得到衍生物 $\mathrm{BCzPh}$ (13)和 BCzTPM (14), 其三线态能级均为 $2.87 \mathrm{eV}$, 掺杂 FIrpic 制备的蓝 光器件, 亮度为 $100 \mathrm{~cd} / \mathrm{m}^{2}$ 时的驱动电压均为 $3.1 \mathrm{~V}$, 同 样条件下用 $\mathrm{mCP}(2)$ 制备的器件驱动电压均为 $3.7 \mathrm{~V}$, 其 中 BCzTPM 制备的蓝光器件最高功率效率 $45.5 \mathrm{~lm} / \mathrm{W}$, 电流效率达到 $44.5 \mathrm{~cd} / \mathrm{A}$, 外量子效率达到 19.6\%. BCzTPM (14) 的 HOMO 为 $-5.26 \mathrm{eV}$, 高于 $\mathrm{mCP}(2)$ 的 HOMO $(-5.80 \mathrm{eV})$, 更有利于空穴的传输, 其单线态与 三线态能级差为 $0.44 \mathrm{eV}$, 低于 $\mathrm{mCP}(2)$ 的 $0.49 \mathrm{eV}$, 从而 降低了驱动电压. 


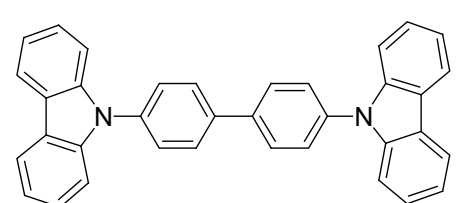

$1 \mathrm{CBP}$

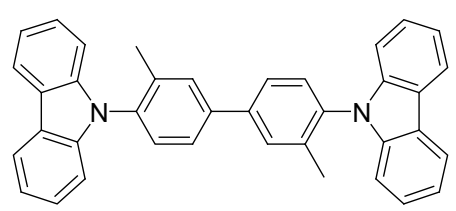

4 o-CDBP

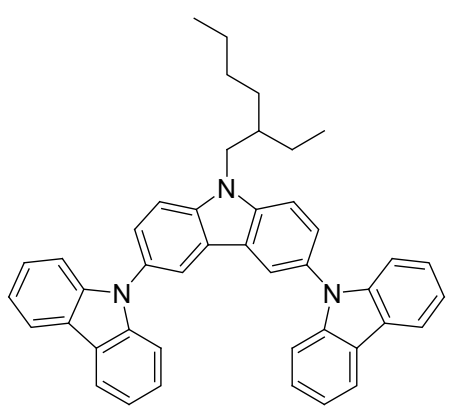

$7 \mathrm{TCz} 1$

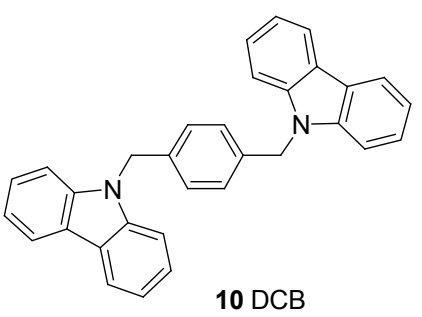

$10 \mathrm{DCB}$

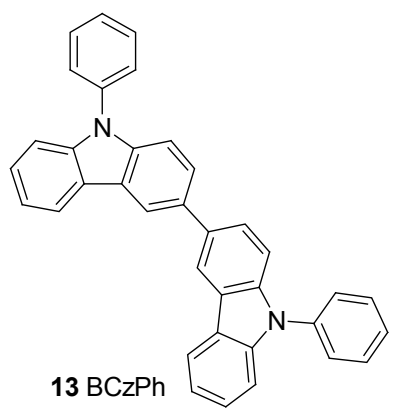<smiles>c1cc(-n2c3ccccc3c3ccccc32)cc(-n2c3ccccc3c3ccccc32)c1</smiles>

$2 \mathrm{mCP}$

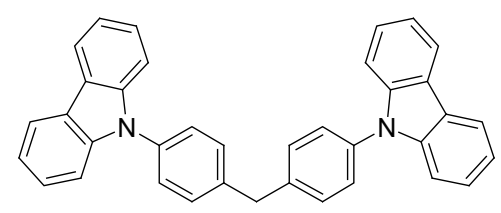

5 CBPM

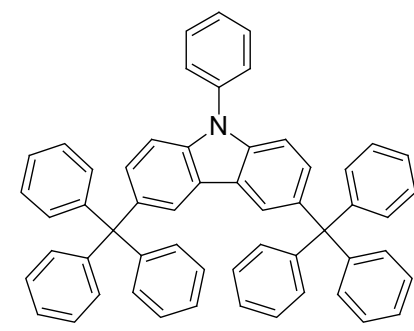

$8 \mathrm{CzC}$

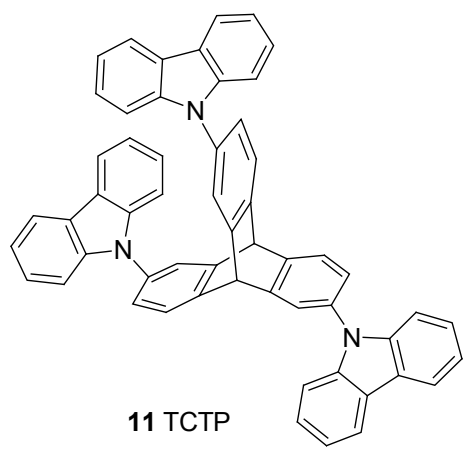

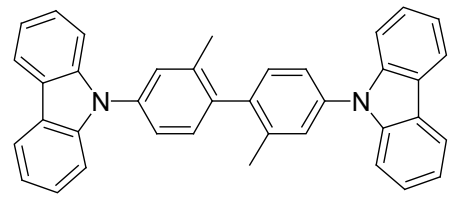

3 CDBP

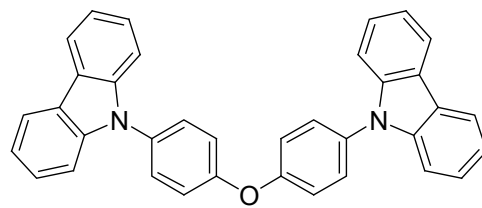

6 CBPE

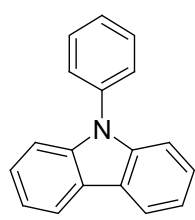

$9 \mathrm{CZPH}$<smiles>c1ccc(-c2ccccc2-c2ccc(-n3c4ccccc4c4ccccc43)cc2)cc1</smiles>

12 4CZPBP
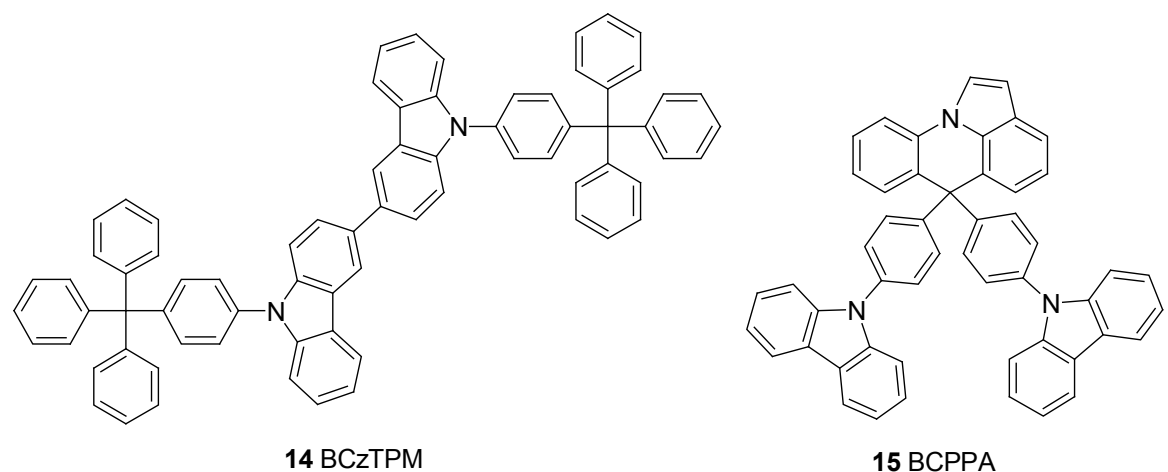

15 BCPPA

图 1 含咔唑基的空穴传输性蓝色磷光主体材料

Figure 1 Hole-transport-type host materials based on carbazole for blue electrophosphorescence

含咔唑的分子还可用作空穴传输性深蓝磷光主体 材料, Park 等[22]合成了 6,6-双[4-(9H-咔唑-9-基)苯基]$6 H$-吡咯 $[3,2,1-d]$ 呋啶(BCPPA, 15), 玻璃态转化温度为 $125{ }^{\circ} \mathrm{C}$, 三线态能级为 $2.97 \mathrm{eV}$, 掺杂客体磷光材料双
[(3,5-二氟-4-氰基苯基)吡啶]吡啶甲酰合铱(FCNIrpic), 应用于深蓝磷光器件, 起亮电压为 $3.5 \mathrm{~V}$, 外量子效率最 高达到 $24.7 \%$, 亮度为 $1000 \mathrm{~cd} / \mathrm{m}^{2}$ 时, 驱动电压为 $7.4 \mathrm{~V}$, 获得电流效率最高达到 $29.9 \mathrm{~cd} / \mathrm{A}$, 功率效率达到 29.3 
$\mathrm{lm} / \mathrm{W}$, 器件的色坐标为 $(0.137,0.182)$.

\section{1 .2 混合型空穴传输性蓝色磷光主体材料}

除咔唑外，还有一些基团如芴、三芳胺也具有空穴 传输性能，也经常用于空穴传输性蓝色磷光主体材料. 在许多情况下, 会通过对这些片段进行组合, 得到混合 型空穴传输性蓝色磷光主体材料.

与咔唑相比, 芴的单线态和三线态能级分裂较大, 因此芴的三线态能级降低到 $2.80 \mathrm{eV}^{[10]}$. 通过对芴分子 结构进行修饰, 使得该类分子具有较高的三线态能级, 同时有良好的热力学稳定性和成膜性能，适合作为蓝色 磷光主体材料. 就分子传输特性而言, 含有咔唑基团的 衍生物一般都具有空穴传输的特性, 而芴也被证明能够 同时传输空穴和电子[23].

Qiao 等 ${ }^{[24]}$ 将咔唑基团通过芴环的 $\mathrm{sp}^{3}$ 杂化的碳原子 连接, 这样分子的共轭程度没有明显增加, 其中 9,9-二 [4-(9-咔唑基)苯基]芴(CPF，16)和 9,9-二[4-(3,6-叔丁基 咔唑基)苯基]芴(TBCPF，17)的三线态能级同样为 2.88 $\mathrm{eV}$, 叔丁基的引入减弱了分子间作用力，阻止了分子聚 集体的形成，从而有效地抑制了磷光染料的三线态激子 的自猝灭. 其中 TBCPF 为主体材料掺杂 FIrpic 的蓝光器 件的电流效率为 $13.0 \mathrm{~cd} / \mathrm{A}$, 要高于未取代叔丁基的母体 $\mathrm{CPF}$ (16)的 $11.4 \mathrm{~cd} / \mathrm{A}$ 的器件电流效率 ${ }^{[24]}$. Shih 等 ${ }^{[25,26]}$ 合 成了芴与咔唑偶联衍生物 CBZ1-F2 (18)及芴与三苯胺偶 联的衍生物三[4-(9-苯基芴基)苯基]胺(TFTPA，19), 其
中 $\mathrm{CBZ} 1-\mathrm{F} 2$ 具有较高的玻璃态转化温度 $108 \sim 231{ }^{\circ} \mathrm{C}$, 三线态能级为 $2.88 \mathrm{eV}$ 左右, CBZ1-F2 掺杂 FIrpic 制备的 蓝光器件的电流效率为 $19.2 \mathrm{~cd} / \mathrm{A}$, 外量子效率为 $10.2 \%$; TFTPA 的玻璃态转化温度高达 $186{ }^{\circ} \mathrm{C}$, 三线态能级为 $2.89 \mathrm{eV}$, 掺杂 FIrpic 的蓝光器件的电流效率为 29.4 $\mathrm{cd} / \mathrm{A}$, 外量子效率达到 $13.1 \%$.

Sasabe 等 ${ }^{[13]}$ 在 3,3'-二咔唑分子的 9,9'-位分别引入三 苯胺基, 得到混合型空穴传输性主体材料 BCzTPA (20). 其三线态能级均为 $2.91 \mathrm{eV}$, 掺杂 FIrpic 制备的蓝光器 件, 亮度为 $100 \mathrm{~cd} / \mathrm{m}^{2}$ 时的驱动电压为 $3.1 \mathrm{~V}$, 最高功率 效率 $39.3 \mathrm{~lm} / \mathrm{W}$, 电流效率达到 $38.2 \mathrm{~cd} / \mathrm{A}$, 外量子效率 达到 $16.6 \%$.

\section{2 含硅的蓝色磷光主体材料}

近年来, OLED 材料已逐渐发展到深蓝色系统，随 着深蓝染料的开发, 要找到合适的主体材料变的越来越 困难. 由于深蓝染料的能隙变大，主体材料的能隙也必 须跟着变大, 才能有效的将能量转移给客体染料. 但应 用到器件时发现，能隙过大容易造成电子或空穴注入的 问题. Thompson 等 ${ }^{[27]}$ 提出电子和空穴直接注入到客体 染料并激发实现发光的概念，主体分子只需要具有高的 三线态能级避免能量回传，同时达到稀释客体染料避免 三线态激子湮灭. 2003 年, Thompson 等 ${ }^{[27,28]}$ 报道的深蓝 磷光主体材料有机硅化合物 UGH 系列(UGH1，21), 该

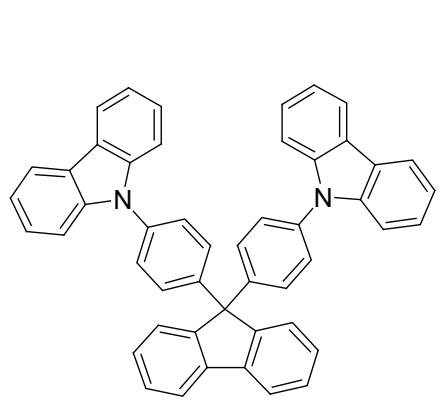

$16 \mathrm{CPF}$

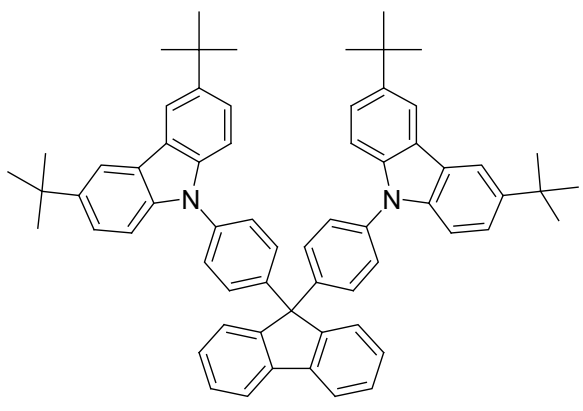

17 TBCPF

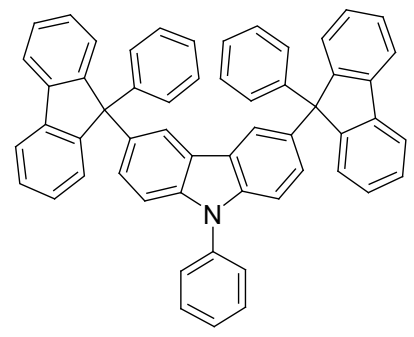

18 CBZ1-F2

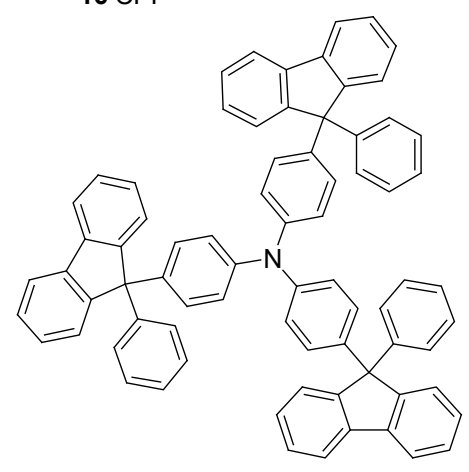

19 TFTPA

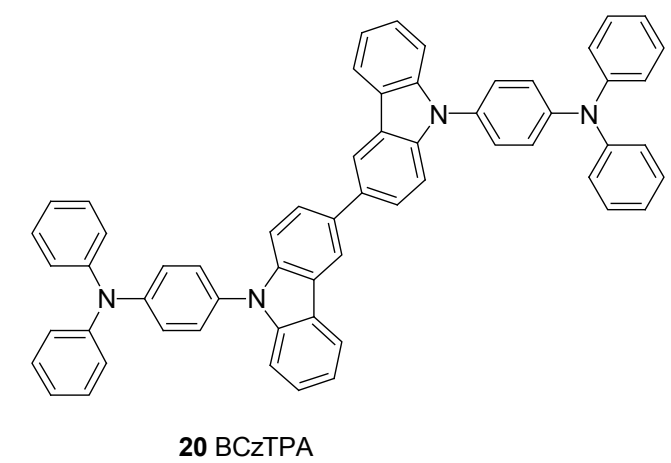

图 2 混合型空穴传输性蓝色磷光主体材料

Figure 2 Hole-transport-type host materials based on mixed groups for blue electrophosphorescence 
系列材料分子结构中使用硅原子将分子中每个苯环都 隔离开, 将共轭度缩小到最低, 所以材料分子的能隙都 很大(约 $4.3 \mathrm{eV}$ ), 也具有很高的三线态能级(约 $3.15 \mathrm{eV}$ ).

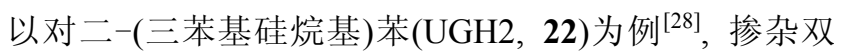
(4',6'-二氟苯基吡啶-N, $\mathrm{C}^{2}$ )-四(1-吡唑基)硼酸合铱(FIr6) 的深蓝光器件的最大外量子效率为 $12.8 \%$, 最大功率效 率为 $15.3 \mathrm{~lm} / \mathrm{W}$. 其中染料 FIr6 的 HOMOs /LUMOs 正好 处于主体材料 UGH2 的 HOMOs 与 LUMOs 之间, 即 FIr6 本身就是所谓的载流子陷阱. 从器件的电压与电流密度 关系看出, 随着 FIr6 含量的增大, 器件的电流密度上升, 起亮电压逐渐降低. 遗憾的是, UGH 系列化合物的热稳 定很差，限制了它们的应用.

Chen 等 ${ }^{[29]}$ 报道了 3,5-二(9-咔唑基)-四苯基硅烷 $(\mathrm{SimCP}, 23)$, 该分子三线态能级为 $2.9 \mathrm{eV}$, 掺杂 FIrpic 的蓝光器件的外量子效率达到 $14.4 \%$, 功率效率为 11.9 $\mathrm{lm} / \mathrm{W}$. 文中还对比了 $\mathrm{mCP}(2)$ 和 $\operatorname{SimCP}(23)$ 作为深蓝磷 光主体材料的性能, 以 $\mathrm{mCP}$ (2) 作为主体材料, 掺杂双 (4,6-二氟苯基吡啶)-5-(吡啶-2-基)- $1 H$-四氮唑合铱 (FIrN4) 器件, 外量子效率为 $5.8 \%$; 当主体换成 $\mathrm{SimCP}$
时, 相同的器件效率提高了 $60 \%$ 以上, 外量子效率达到 9.4\%. 作者认为 $\mathrm{SimCP}$ 相对于 $\mathrm{mCP}$ 具有高的玻璃态转 化温度, 大的刚性分子结构防止了器件中三线态激子的 湮灭, 同时四苯基硅烷的引入提高了器件的电子传输能 力, 从而使载流子得到有效的复合, 因此器件效率大大 提高.

$\mathrm{Wu}$ 等 ${ }^{[30]}$ 直接将三苯基硅烷接在咔唑的 3,6-位，主 体材料 9-(4-叔丁基苯基)-3,6-二(三苯基硅基)咔唑(CzSi, 24)三线态能级为 $3.02 \mathrm{eV}$, 掺杂 FIrpic 的蓝光器件的外 量子效率达到 $16.7 \%$, 电流效率为 $30.6 \mathrm{~cd} / \mathrm{A}$, 功率效率 为 $26.7 \mathrm{~lm} / \mathrm{W}$. Tsuboi 等 ${ }^{[31]}$ 合成了二[3,5-二(9-咔唑基)苯 基]二苯基硅烷 $(\mathrm{SimCP} 2,25)$ 作为主体材料, 掺杂 FIrpic 的蓝光器件的外量子效率达到 $17.7 \%$, 功率效率为 24.2 $\mathrm{lm} / \mathrm{W} . \mathrm{Lu}$ 等 ${ }^{[32]}$ 报道了蓝色磷光主体分子二[4-(9-咔唑基) 苯基]二甲基硅烷(DMSiCBP, 26)和二[4-(9-咔唑基)苯基] 二苯基硅烷(DPSiCBP，27). 该类分子同样保持高的三 线态能级, 约 $3.02 \mathrm{eV}$, 掺杂 FIrpic 的蓝光器件的外量子 效率达到 $3.5 \%$, 电流效率为 $6.5 \mathrm{~cd} / \mathrm{A}$. Lin 等 ${ }^{[33]}$ 报道了 4,4'-二(三苯基硅烷基)联苯(BSB，28), 掺杂 FIrpic 的蓝

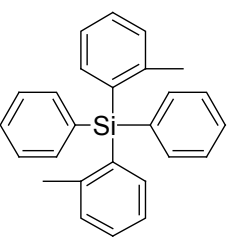

$21 \mathrm{UGH} 1$

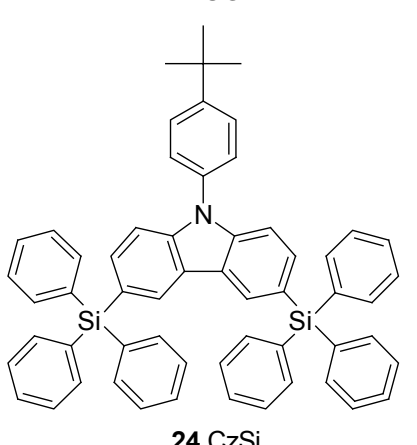

$24 \mathrm{CzSi}$

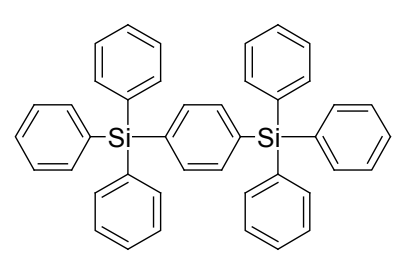

22 UGH2

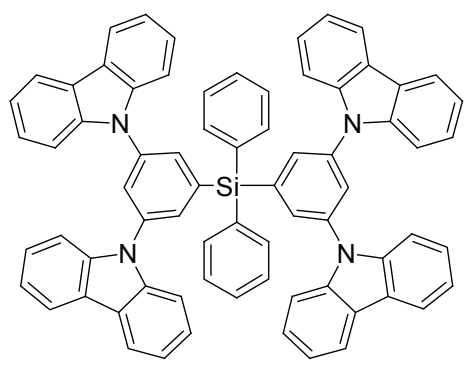

25 SimCP2

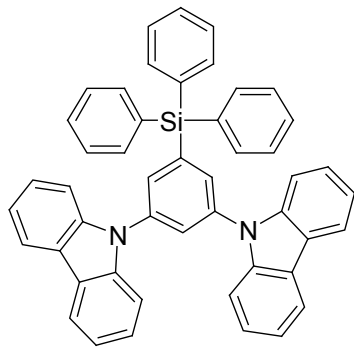

$23 \operatorname{Sim} C P$

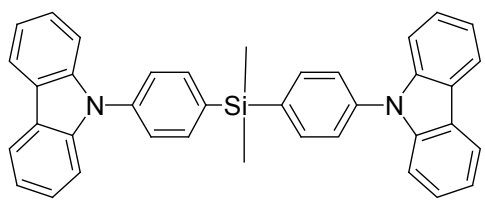

26 DMSiCBP

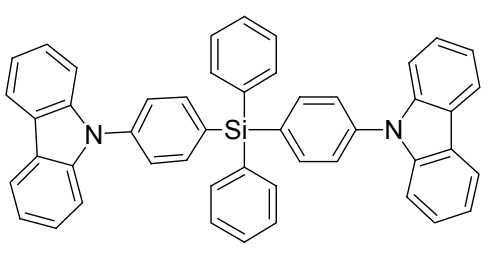

27 DPSiCBP
$29 \mathrm{SitCz}$

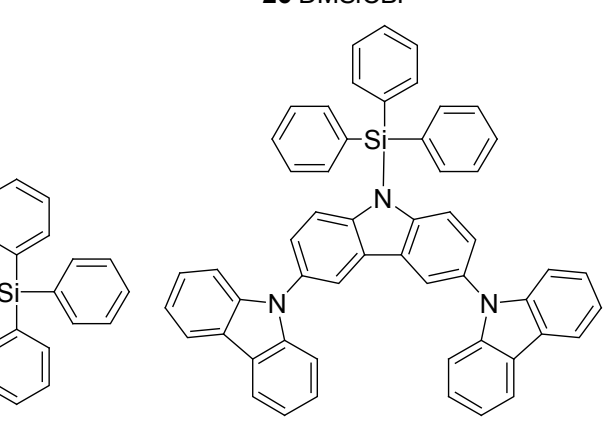

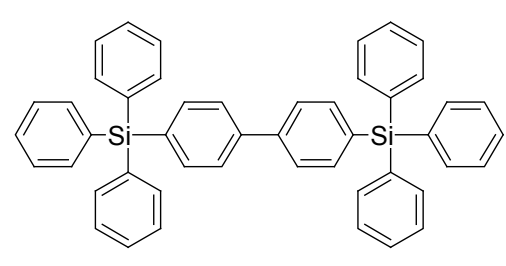

28 BSB
图 3 含硅的蓝色磷光主体材料

Figure 3 Host materials based on silane for blue electrophosphorescence 
光器件的外量子效率达到 $14.3 \%$, 电流效率为 $33.5 \mathrm{~cd} / \mathrm{A}$. Hong 等 ${ }^{[34]}$ 报道了蓝色磷光主体分子三苯基硅基三咔唑 $(\mathrm{SitCz}, 29)$, 玻璃态转化温度为 $170{ }^{\circ} \mathrm{C}$, 掺杂 FIrpic 的蓝 光器件的外量子效率达到 $27.2 \%$, 功率效率为 36.5 $\mathrm{lm} / \mathrm{W}$, 电流效率为 $46.3 \mathrm{~cd} / \mathrm{A}$, 亮度为 $100 \mathrm{~cd} / \mathrm{m}^{2}$ 时, 色坐 标为 $(0.13,0.29)$.

\section{3 电子传输性蓝色磷光主体材料}

在一般的 OLED 器件中, 常见的情况往往是空穴在 发光层的量多于电子在发光层的量, 从而导致较低的复 合几率, 这归处于大多数 OLED 传输材料中, 空穴比电 子有更好的传输性能. 因此, 采用电子传输性能好的材 料也是提高器件性能的方法之一. 显然, 电子传输性主 体材料应用于 PhOLED, 同样有利于提高蓝色磷光器件 的性能

含膦氧基化合物常被用于作为电子传输性主体材 料, Burrows 等 ${ }^{[35,36]}$ 设计并合成了电子传输性主体材料 4,4'-二(二苯基磷氧基)联苯(PO1，30)和 2,7-二(二苯磷氧 基)-9,9'-二甲基芴(PO6，31)，该类分子的三线态能级与 FIrpic 相当, 掺杂 FIrpic 的蓝光器件的最高外量子效率 为 $8.1 \%$, 功率效率为 $21.5 \mathrm{~lm} / \mathrm{W}$. 由于其良好的电子传 输性能, 器件具有较低的驱动电压, 因此表现出较高的 功率效率. 为了提高三线态能级, 合成了类似分子 $2,8-$ 二(二苯磷氧基)二苯呋喃(POF, 32 $)^{[37]}$, 其三线态能级 $3.12 \mathrm{eV}$, 使用该主体材料制得的器件的外量子效率提 高到 $10.4 \%$, 电流效率为 $26.9 \mathrm{~cd} / \mathrm{A}$, 功率效率为 25.9 $\mathrm{lm} / \mathrm{W}$.

Chen 等 ${ }^{[38]}$ 合成的多聚苯树枝基团的啞二唑主体材 料 2,5-二[4-(2,3,4,5-四苯基)苯基]苯基-[1,3,4]-噁二唑 $(\mathrm{BTPO}, 33)$, 掺杂 FIrpic 的蓝光器件的外量子效率为
$6.2 \%$, 电流效率为 $11.9 \mathrm{~cd} / \mathrm{A}$.

Fukagawa 等 ${ }^{[39]}$ 将吡啶基团和吲哚结合, 合成了 2,2二(4-吡啶并吲哚基-9-苯基)金刚烷(Ad-Pd, 34). 分子中 吡啶并吲哚基团的三线态能级类似咔唑基团，分子的三 线态能级达到 $2.97 \mathrm{eV}$, 并且具有强的电子传输能力. 两 分子之间通过金刚烷进行连接，使得分子的玻璃态转化 温度达到 $181{ }^{\circ} \mathrm{C}$, 掺杂 FIr6 的深蓝光器件的最大量子效 率为 $19 \%$, 最大功率效率为 $33 \mathrm{~lm} / \mathrm{W}$.

\section{4 双极性蓝色磷光主体材料}

前面介绍的主体材料大多数只具备单载流子传输 特性, 而载流子的注入及传输是影响 OLED 器件性能的 关键因素之一. 如果在发光层中, 电子和空穴浓度不匹 配, 不仅会降低器件的效率, 还将加速器件的老化. 双 极主体材料的使用，不仅能使载流子在发光层中平衡， 同时还可能实现单功能层器件, 从而简化器件的制备工 艺. 目前报道的双极主体材料，从分子设计角度通常将 传输电子基团与传输空穴的基团进行偶联就可以得到. 但是，当缺电子基团和富电子基团直接或间隔 $\pi$ 体系相 连接, 容易形成分子内电荷转移, 同时将大大降低分子 的三线态能级. 因此，开发具有双极传输特性的蓝色磷 光主体材料是很多研究小组一直追求的目标 ${ }^{[7]}$.

日本的 Kido 小组长期从事电子传输材料的开发, 主要是以吡啶作为电子传输单元进行分子设计. $\mathrm{Su}$ 等 ${ }^{[40]}$ 在此基础上采用咔唑作为空穴传输基团，吡啶作为电子 传输基团，中间通过苯环的间位进行连接，制备了双极 性蓝色磷光主体材料 2,6-二[3-(9-咔唑基)苯基]吡啶 (26DCzPPy，35)和 3,5-二[3-(9-咔唑基)苯基]吡啶 (35DCzPPy, 36). 该类分子的三线态能级均为 $2.71 \mathrm{eV}$, 掺杂 FIrpic 的蓝光器件在 $100 \mathrm{~cd} / \mathrm{m}^{2}$ 的亮度下, 功率效率
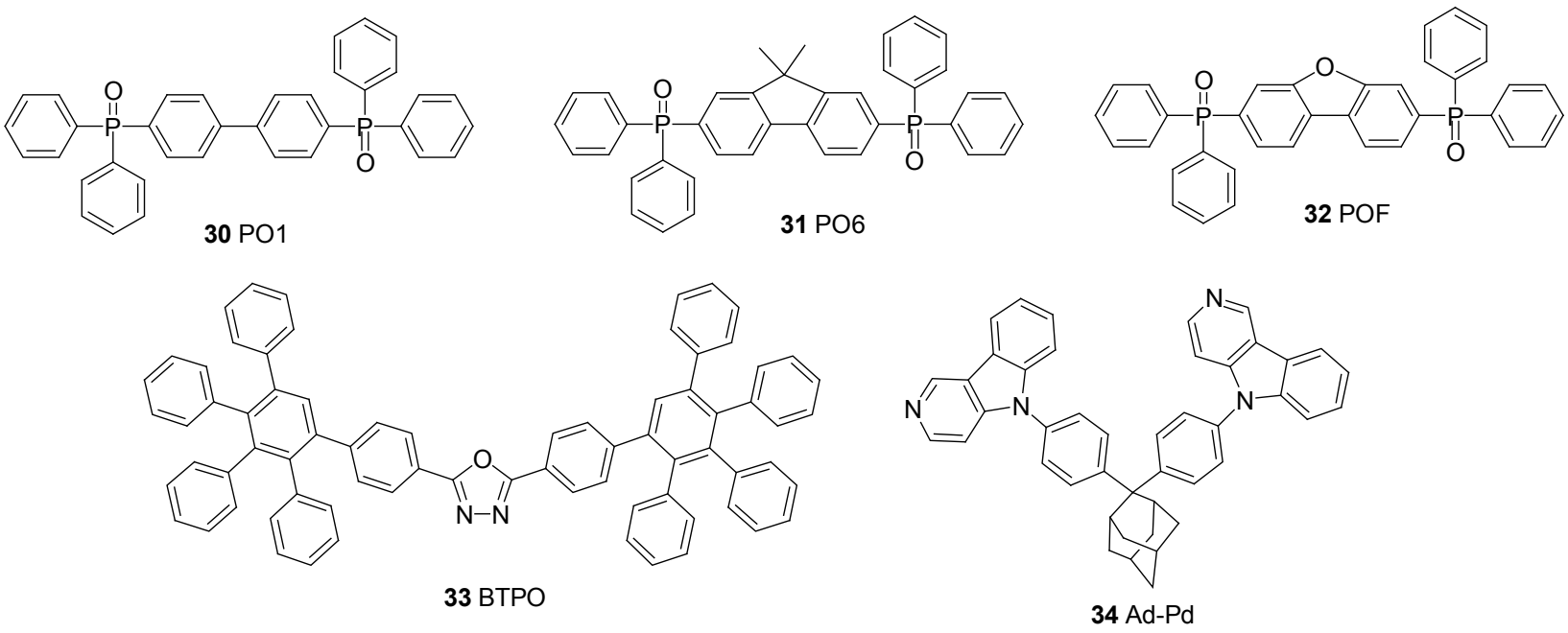

图 4 电子传输性蓝色磷光主体材料

Figure 4 Electron-transport-type host materials for blue electrophosphorescence 
为 $46 \mathrm{~lm} / \mathrm{W}$, 外量子效率达到 $24.3 \%$; 在 $1000 \mathrm{~cd} / \mathrm{m}^{2}$ 的亮 度下, 功率效率仍然达到 $34.5 \mathrm{~lm} / \mathrm{W}$, 外量子效率为 $22.6 \%$. 值得注意的是, Kido 小组在蓝色磷光器件中使 用的空穴传输材料和电子传输材料都具有高的三线态 能级和高的载流子迁移率, 因此载流子能高效的被限制 在发光层复合实现发光, 从而大大提高了器件效率. 同 时, 他们还认为采用高三线态能级的空穴和电子传输层 对器件效率的贡献大于采用高电子迁移率材料.

Rothmann 等 ${ }^{[41]}$ 采用三嗪基团作为电子传输核心, 制备了 TRZ 系列主体材料, 其中二[4,6-二(3-苯基咔唑 基)-1,3,5-三嗪基]间苯二醚(TAZ7，39)具有最高的玻璃 态转化温度为 $170{ }^{\circ} \mathrm{C}$. 以 2,4-二(9-咔唑基)-6-苯氧基1,3,5-三嗪(TAZ2, 38) 为例, 掺杂 FIrpic 的蓝光器件的外 量子效率为 $10.2 \%$. Huang 等 ${ }^{[42]}$ 以 $1,3,5$-三嗪为电子传输 核心, 通过用咔唑基、二苯胺基、苯基、噻吩基对 2,4,6位进行不对称取代, 得到系列主体材料, 这些材料的三 线态能级都在 $2.65 \sim 2.96 \mathrm{eV}$, 其中 2-苯基-4,6-二(9-咔 唑)基-1,3,5-三嗪(PhDCzT, 40)掺杂 FIrpic 的蓝光器件的 最高外量子效率为 $9.8 \%$, 功率效率为 $20.0 \mathrm{~lm} / \mathrm{W}$, 电流 效率为 $20.9 \mathrm{~cd} / \mathrm{A}$, 器件性能优于对称取代的 TAZ1 (37). $\mathrm{Su}$ 等 ${ }^{[43]}$ 在三溙的 2,4,6-位引入三个苯基咔唑, 得到主体 材料 TCPZ (41), 其三线态能级为 $2.52 \mathrm{eV}$, 低于 FIrpic 的三线态能级 $(2.62 \mathrm{eV})$, 制得的蓝色磷光器件在亮度为 $100 \mathrm{~cd} / \mathrm{m}^{2}$ 时, 外量子效率为 $10.1 \%$, 功率效率达到 25.4 $\mathrm{lm} / \mathrm{W}$, 驱动电压仅为 $3.74 \mathrm{~V}$. 这是由于其单线态能级与 三线态能级非常接近, 仅为 $0.14 \mathrm{eV}$, 降低了驱动电压.

具有传电子功能的膦氧基团常常被用于蓝色磷光 主体材料的分子设计中. 在 $\pi$ 共轭体系中引入膦氧基团, 能打断分子的共轭体系, 使分子保持很高的三线态能 级. 在双极主体材料分子的研究中, 很多研究小组将传 输空穴的咔唑基团和传输电子的膦氧基团连接在一起, 该类分子一般具有很高的三线态能级，同时具有空穴和 电子传输能力. 采用该类分子作为主体材料, 还能有效 地降低器件的驱动电压, 同时使器件效率大大提高.

Burrows 课题组 ${ }^{[44]} 2008$ 年发表了研究膦氧基团作为 蓝色磷光主体材料的研究论文, 其中材料 3,6-二(二苯基 膦氧基)-9-苯基咔唑(PO9, 也就是 PPO2, 43)三线态能级 为 $3.02 \mathrm{eV}$, 掺杂 FIrpic 的蓝光器件外量子效率约在 6 $8 \%$. Chou 等 ${ }^{[45]}$ 将膦氧基团通过苆环的 $\mathrm{sp}^{3}$ 杂化的碳原子 连接, 辅助以二芳胺基团, 制得了 2,7-二(二苯基膦氧 基)-9-[4-( $N, N$-二苯胺基)苯基]-9-苯基芴(POAPF, 44). 该 分子三线态能级为 $2.75 \mathrm{eV}, \mathrm{HOMO}$ 能级为 $-5.26 \mathrm{eV}$, 同时空穴和电子的单载流子器件也说明了其双极传输 特性, 掺杂 FIrpic 的蓝光器件起亮电压为 $2.5 \mathrm{~V}\left(1 \mathrm{~cd} / \mathrm{m}^{2}\right)$, 外量子效率为 $20.6 \%$, 最高电流效率为 $35.4 \mathrm{~cd} / \mathrm{A}$, 功率
效率为 $36.7 \mathrm{~lm} / \mathrm{W}$. 文中报道膦氧基团具有良好的电子 注入的能力, 作者在发光层后直接蒸镀了 $100 \mathrm{~nm}$ 的 $\mathrm{Al}$ 电极, 器件的外量子效率高达 $17.2 \%$. Lee 等 ${ }^{[46,47]}$ 先后发 表了一系列用于深蓝磷光双极传输主体材料, 3-(二苯基 膦氧基)-9-苯基-9H-咔唑(PPO1, 42), PPO2 (43), 3-(二苯 基膦氧基)-9-[4-(二苯基膦氧基)苯基]-9-咔唑(PPO21, 45), 3-(二苯基膦硫基)-9-[4-(二苯基膦硫基)苯基]-9-咔唑 (PPS21，46), 掺杂 FCNIrpic 的蓝光器件色坐标为 $(0.14$, 0.15), 最高外量子效率高达 $19.2 \%$. Cheng 等 ${ }^{[48]}$ 在 CBP 分子结构的基础引入膦氧基团, 制备了双极传输主体材 料二[4-( $N$-咔唑基)苯基]苯基膦氧( $\mathrm{BCPO}, 47)$. 该分子的 三线态能级为 $3.01 \mathrm{eV}$, 大于 $\mathrm{CBP}$ 的 $2.56 \mathrm{eV}$; 玻璃态转 化温度为 $137{ }^{\circ} \mathrm{C}$, 远大于 $\mathrm{CBP}$ 的 $62{ }^{\circ} \mathrm{C}$. 该主体材料掺 杂 FIrpic 的蓝光器件起亮电压为 $2.8 \mathrm{~V}\left(1 \mathrm{~cd} / \mathrm{m}^{2}\right)$, 最大外 量子效率为 $23.5 \%$, 最高电流效率为 $45.1 \mathrm{~cd} / \mathrm{A}$, 功率效 率为 $40.6 \mathrm{~lm} / \mathrm{W}$; 掺杂 FIr6 的深蓝磷光器件起亮电压为 $3.0 \mathrm{~V}\left(1 \mathrm{~cd} / \mathrm{m}^{2}\right)$, 最大外量子效率为 $19.8 \%$, 最高电流效 率为 $36.8 \mathrm{~cd} / \mathrm{A}$, 功率效率为 $33.1 \mathrm{~lm} / \mathrm{W}$.

近期 $\mathrm{Xu}$ 等 ${ }^{[49,50]}$ 在 9,9-二苯基芴分子的两个苯环上 分别引入传输电子的膦氧基团和传输空穴的三苯胺、咔 唑基团, 得到系列双极传输蓝色磷光主体材料 9ArPAFSPO, 三线态能级均大于 $3.0 \mathrm{eV}$, 玻璃态转化温 度均大于 $118{ }^{\circ} \mathrm{C}$, 其中 9-[4-\{9-[4-(二苯膦氧基)苯基]$9 \mathrm{H}$ - 芴-9-基 $\}$ 苯基] $-9 H$-咔唑(9CzPAFSPO, 48), 掺杂 FIrpic 的蓝色磷光器件, 最高功率效率 $35.6 \mathrm{~lm} / \mathrm{W}$, 电流 效率 $35.5 \mathrm{~cd} / \mathrm{A}$, 最大外量子效率为 $14.4 \%$, 在亮度为 $200 \mathrm{~cd} / \mathrm{m}^{2}$ 时, 最低驱动电压低于 $4.1 \mathrm{~V}$. 他们还对二苯 呋喃分子进行修饰, 在环上分别引入咔唑和膦氧基团, 得到多个主体材料分子 DBFxPOCzn ${ }^{[51]}$, 这些分子的三 线态能级为 $2.88 \sim 2.91 \mathrm{eV}$, 玻璃态转化温度均大于 100 ${ }^{\circ} \mathrm{C}$. 与前面提到的电子传输性主体材料 POF (32)相比, 该类分子能隙大大降低, 咔唑的引入有利于空穴注入, 分子在保持较高的三线态能级的同时, 单线态能级降 低, 从而有利于降低驱动电压. 其中 2-咔唑基-6-(二苯 膦氧基)二苯呋喃(DBFSPOCz, 49)掺杂 FIrpic 的蓝光器 件, 亮度为 $1000 \mathrm{~cd} / \mathrm{m}^{2}$ 驱动电压低于 $3.6 \mathrm{~V}$, 外量子效率 达到 $27 \%$, 最高电流效率为 $27 \mathrm{~cd} / \mathrm{A}$, 功率效率为 45 $1 \mathrm{~m} / \mathrm{W}$.

$\mathrm{Ma}$ 等 ${ }^{[52]}$ 通过在四苯基硅分子上引入传输电子的膦 氧基团和传输空穴的咔唑基团得到 4-二苯基膦氧-4'-9H咔唑-9-基-四苯基硅烷(CSPO, 50), 玻璃态转化温度达 到 $140{ }^{\circ} \mathrm{C}$, 三线态能级为 $2.97 \mathrm{eV}$, 掺杂 FIrpic 的蓝色磷 光器件, 最大外量子效率为 $27.5 \%$, 电流效率为 49.4 $\mathrm{cd} / \mathrm{A}$. 
<smiles>[X]c1cc(-c2cccc(-n3c4ccccc4c4ccccc43)c2)ncc1-c1cccc(-n2c3ccccc3c3ccccc32)c1</smiles>

35 26DCzPPy $X=\mathrm{CH}, \mathrm{Y}=\mathrm{N}$

36 35DCzPPy $X=\mathrm{N}, \mathrm{Y}=\mathrm{CH}$<smiles>Cc1ccc2c(c1)c1ccccc1n2-c1nc(Oc2cccc(Oc3nc(-n4c5ccccc5c5cc(C)ccc54)nc(-n4c5ccccc5c5cc(C)ccc54)n3)c2)nc(-n2c3ccccc3c3ccccc32)n1</smiles>

39 TRZ7

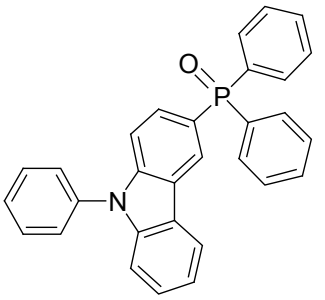

$42 \mathrm{PPO} 1$

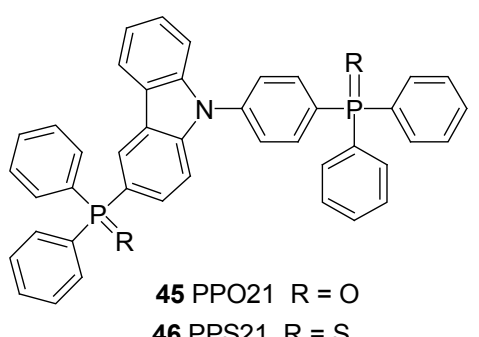

$46 \mathrm{PPS} 21 \mathrm{R}=\mathrm{S}$

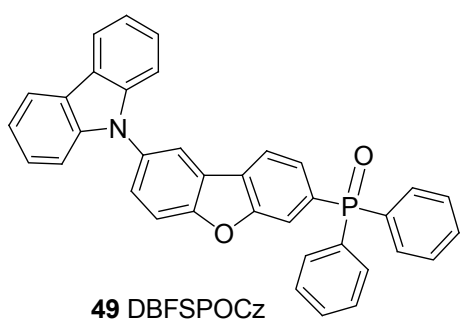

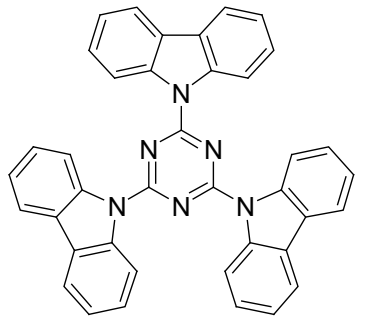

37 TRZ1

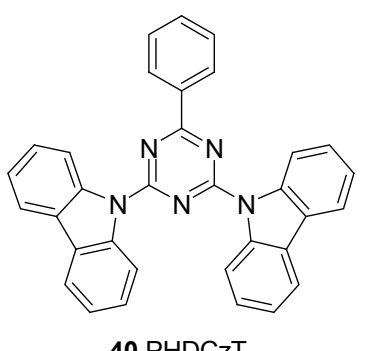

40 PHDCzT<smiles>c1ccc(Oc2nc(-n3c4ccccc4c4ccccc43)nc(-n3c4ccccc4c4ccccc43)n2)cc1</smiles>

38 TRZ2

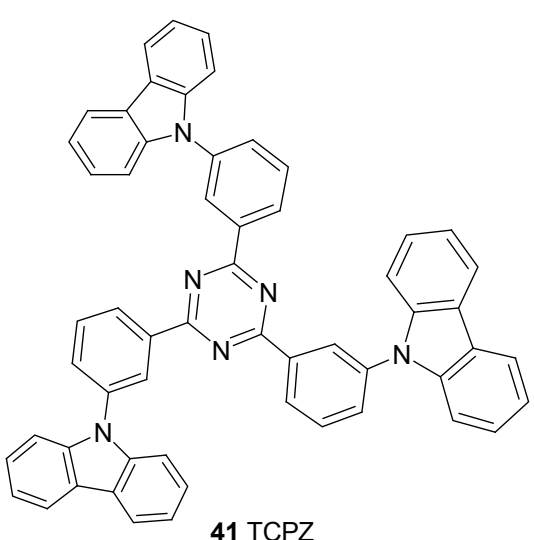

$41 \mathrm{TCPZ}$

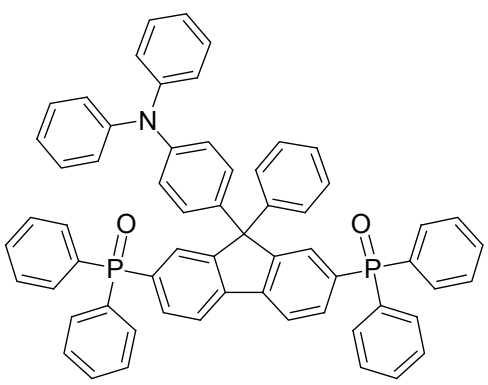

44 POAPF

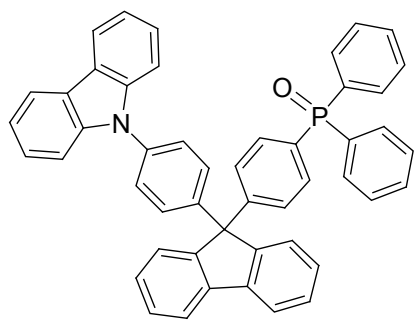

48 9CzFSPO<smiles>O=P(c1ccccc1)(c1ccccc1)c1ccc([Si](c2ccccc2)(c2ccccc2)c2ccc(-n3c4ccccc4c4cc(-n5c6ccccc6c6ccccc65)ccc43)cc2)cc1</smiles>

50 DCSPO

图 5 双极蓝色磷光主体材料

Figure 5 Bipolar host materials for blue electrophosphorescence 


\section{5 用于湿法制膜的蓝色磷光主体材料}

近年来, 可湿法制备的小分子电致磷光器件由于其 更高的能量利用效率得到了人们更多的关注. 小分子材 料大多采用真空蒸镀成膜, 易于制备较为复杂的多层结 构器件以实现载流子注入传输平衡, 从而得到高效率的 器件. 但是, 真空蒸镀过程中材料的利用率很低; 同时 器件制备工艺非常复杂, 成本较高, 并且很难实现大面 积高解析度的显示面板 ${ }^{[53]}$. 湿法制膜, 相对于真空蒸镀 而言, 工艺简单，设备低廉，成本低，可制备大面积发 光层. 另外, 对于掺杂结构的器件, 真空蒸镀通常要采 用双蒸发源或多蒸发源共蒸的方法实现, 材料的掺杂比 例很难控制, 而采用湿法制备时可以通过调控溶液中材 料的浓度从而精确控制薄膜中材料的掺杂比例, 这对于 制备多元掺杂的超低掺杂浓度薄膜尤为方便. 因此, 开 发高性能的可湿法制备的有机小分子电致发光器件意 义重大.

由于目前常用的可溶液加工的小分子主体材料, 如 CBP (1)等, 其三线态能级 $(2.56 \mathrm{eV})$ 都低于常见的蓝色 磷光染料(如 FIrpic, $2.62 \mathrm{eV}$ ), 很难湿法制备高效率的小 分子蓝色磷光器件. MCP (2) 和硅类 UGH 系列衍生物都 是真空蒸镀器件中常用的具有高三线态能级的蓝色磷 光主体材料, 它们的三线态能级 $(>2.9 \mathrm{eV})$ 都高于常用 的蓝色磷光染料, 但这些宽带隙小分子主体材料的玻璃 态转化温度都非常低(如 $\mathrm{mCP}, 65{ }^{\circ} \mathrm{C}$; UGH1, $26{ }^{\circ} \mathrm{C}$ ), 而 且湿法制备的薄膜易结晶. 因此它们也并不是理想的可 溶液加工的小分子蓝色磷光主体材料. 实际上, 目前报 道的适合湿法制备的小分子蓝色磷光主体材料不多.

空穴传输性主体材料可用于湿法制备小分子蓝色 磷光器件. Hou 等 ${ }^{[54]}$ 采用前面提到的主体材料 TBCPF (17), 该分子的玻璃化转变温度高达 $212{ }^{\circ} \mathrm{C}$, 三线态能 级为 $2.88 \mathrm{eV}$, 同时分子含有的叔丁基使其易溶于许多 常规溶剂. 该主体材料掺杂 FIrpic 湿法制备了高效率的 单层小分子蓝色磷光器件, 器件最大电流效率达 14.2 $\mathrm{cd} / \mathrm{A}$, 最大外量子效率为 $6.7 \%$, 最大亮度为 25300 $\mathrm{cd} / \mathrm{m}^{2}$. Ye 等 ${ }^{[55,56]}$ 报道了基于芴的可湿法制膜的蓝色磷 光主体材料 4,4'-[9,9'-(1,3-苯基)双( $9 H$-芴-9,9-二基)]双 $(N, N$-二苯基胺)(DTPAFB, 51), 1,3-双 \{9-[4-(9H-咔唑-9基)苯基]-9H-芴-9-基 $\}$ 苯(DCPFB，52)，1,3-双 \{9-[4-(3,6二叔丁基-9H-咔唑-9-基)苯基]-9H-芴-9-基 $\}$ 苯(DTCPFB, 53). 该类主体具有良好的热稳定性和成膜性能, 湿法制 膜掺杂 $30 \%$ FIrpic 的蓝光器件的最高电流效率为 25.6 $\mathrm{cd} / \mathrm{A}$, 最大功率效率为 $12.3 \mathrm{~lm} / \mathrm{W}$, 色坐标为 $(0.141$, $0.229)$.

Jiang 等 ${ }^{[57,58]}$ 报道了在苯环的 $1,3,5$-位分别引入咔 唑、间三苯基基团或三咔唑基团, 得到星状蓝色磷光主
体材料 DQC (54), PTC (55)和 Cz-TCB (56), 该类主体材 料掺杂 FIrpic 客体染料采用湿法制备的蓝色磷光器件, 最大亮度达到 $25500 \mathrm{~cd} / \mathrm{m}^{2}$, 最大电流效率达到 25.7 $\mathrm{cd} / \mathrm{A}$, 最大外量子效率达到 $11.9 \%$. 基于咔唑基团的蓝 色磷光主体材料 BCC-36 (57), BTCC-36 (58), BCC-27 (59)和 BTCC-27 (60), 该类分子具有很高的三线态能级 $(>2.90 \mathrm{eV})$, 良好热稳定性和湿法成膜性能，该类主体 材料掺杂 FIrpic 客体染料采用湿法制备的蓝色磷光器 件, 器件的起亮电压较低, 约为 $4.0 \mathrm{eV}$, 最高亮度 27300 $\mathrm{cd} / \mathrm{m}^{2}$, 最高电流效率为 $27.2 \mathrm{~cd} / \mathrm{A}$, 最大外量子效率达 $14.0 \%$; 掺杂 FIr6 客体染料的深蓝色磷光器件, 起亮电 压约为 $5.0 \mathrm{~V}$, 最高亮度为 $14500 \mathrm{~cd} / \mathrm{m}^{2}$, 最高电流效率 为 $11.5 \mathrm{~cd} / \mathrm{A}$, 最大外量子效率达 $6.8 \%{ }^{[59]}$.

双极性主体材料也可以通过湿法成膜得到高效率 蓝色磷光器件. Jiang 等 ${ }^{[00]}$ 设计并合成了基于咔唑和膦氧 基团的具有双极传输特性的蓝色磷光主体材料 $\mathrm{CzPO} 1$ (61) 和 $\mathrm{CzPO} 2$ (62). 研究表明该类分子具有较高的三线 态能级 $(>2.81 \mathrm{eV})$, 良好热稳定性和湿法成膜性能, 以 该类主体材料掺杂 FIrpic 客体染料采用湿法制备了蓝色 磷光单层器件和双层器件. 其中以 $\mathrm{CzPO} 2$ 为主体的单 层器件的最大电流效率为 $10.2 \mathrm{~cd} / \mathrm{A}$, 最大外量子效率达 $5.4 \%$; 双层器件的最大电流效率为 $23.6 \mathrm{~cd} / \mathrm{A}$, 最大外量 子效率达 $12.2 \%$. Yang 等 ${ }^{[61]}$ 报道在四苯基硅分子的两个 苯基上分别引入空穴传输基团二苯胺和电子传输基团 1,2,4-三坐, 得到 \{4-[ \{4-[5-(4-叔丁基苯基)-4-苯基-4H1,2,4-三唑-3-基]苯基 $\}$ (二苯基)硅基]苯基 $\}$ 二苯胺 $(p$ TAZSiTPA, 63), 掺杂 FIrpic 客体染料, 采用湿法制备的 蓝色磷光单层器件, 电流效率达到 $23.7 \mathrm{~cd} / \mathrm{A}$, 功率效率 达到 $11.8 \mathrm{~lm} / \mathrm{W}$, 最大外量子效率达到 $11.2 \%$, 色坐标为 $(0.15,0.32)$. Lee 等 ${ }^{[62]}$ 报道了基于苯并呋喃吡啶的双极蓝 色磷光主体材料 3-[3-(咔唑-9-基)苯基]苯并呋喃[2,3-b] 吡啶(PCz-BFP, 64). 作者对比了该主体材料掺杂 FIrpic 客体染料的真空蒸镀器件和湿法制备器件的性能. 在亮 度为 $1000 \mathrm{~cd} / \mathrm{m}^{2}$, 湿法制备器件的外量子效率为 $17.6 \%$, 略低于真空蒸镀器件的 $20 \%$; 但是驱动电压为 $5.4 \mathrm{~V}$, 比 真空蒸镀器件的 $7.1 \mathrm{~V}$ 要低.

\section{2 结论与展望}

磷光器件由于能充分利用单线态和三线态激子, 大 大提高了器件的效率, 有机小分子电致磷光材料与器件 是目前研究领域的一个热点. 迄今为止, 绿色和红色磷 光器件已经实现高的量子效率, 而稳定、高效的蓝色磷 光器件仍然是发展 PhOLEDs 的短板. 作为影响蓝色磷 光器件性能的关键因素, 性能优良的蓝色磷光主体材料 的发展仍然值得期待. 在前面的论述中, 我们详细介绍 


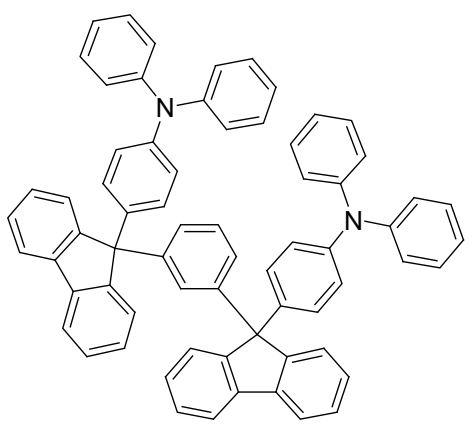

51 DTPAFB<smiles>c1ccc(-c2cc(-c3ccccc3)cc(-c3cc(-c4cc(-c5ccccc5)cc(-c5ccccc5)c4)cc(-n4c5ccccc5c5ccccc54)c3)c2)cc1</smiles>

$55 \mathrm{DQC}$

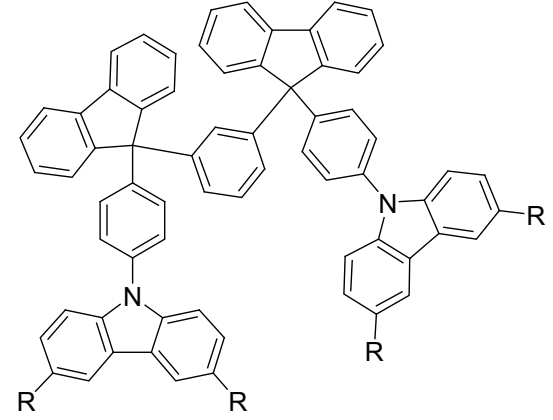

52 DCPFB $\quad \mathrm{R}=\mathrm{H}$ 53 DTCPFB $R=t-B u$<smiles>c1ccc(-c2cc(-c3ccccc3)cc(-c3cc(-n4c5ccccc5c5ccccc54)cc(-n4c5ccccc5c5ccccc54)c3)c2)cc1</smiles>

54 PTC<smiles>CC(C)(C)c1ccc2[nH]c3ccc(C(C)(C)C)cc3c2c1</smiles><smiles>CC(C)(C)c1ccc2c(c1)c1cc(N)ccc1n2-c1cc(-n2c3ccc(N)cc3c3cc(-n4c5ccc(C(C)(C)C)cc5c5cc(C(C)(C)C)ccc54)ccc32)cc(-n2c3ccc(-n4c5ccc(C(C)(C)C)cc5c5cc(C(C)(C)C)ccc54)cc3c3cc(-n4c5ccc(C(C)(C)C)cc5c5cc(C(C)(C)C)ccc54)ccc32)c1</smiles>

$56 \mathrm{Cz}-\mathrm{TCB}$<smiles>[R]c1ccc2c(c1)c1cc([R])ccc1n2-c1ccc2c(c1)c1cc(-n3c4ccc([R])cc4c4cc([R])ccc43)ccc1n2-c1ccccc1</smiles>

57 BCC-36 $\mathrm{R}=\mathrm{H}$ 58 BTCC-36 $\mathrm{R}=t-\mathrm{Bu}$<smiles>[R]c1ccc2c(c1)c1cc([R])ccc1n2-c1ccc2c3ccc([R])cc3n(-c3ccccc3)c2c1</smiles>

$59 \mathrm{BCC}-27 \quad \mathrm{R}=\mathrm{H}$

$60 \mathrm{BTCC}-27 \mathrm{R}=t-\mathrm{Bu}$

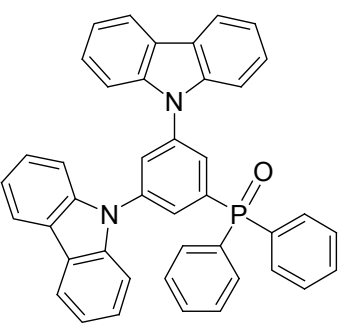

$61 \mathrm{CzPO} 1$<smiles>O=P(c1ccccc1)(c1ccccc1)c1cc(-n2c3ccccc3c3ccccc32)cc(P(=O)(c2ccccc2)c2ccccc2)c1</smiles>

$62 \mathrm{CzPO} 2$<smiles>CC(C)(C)c1ccc(-c2nnc(-c3ccc([Si](c4ccccc4)(c4ccccc4)c4ccc(N(c5ccccc5)c5ccccc5)cc4)cc3)n2-c2ccccc2)cc1</smiles>

$63 p$-TAZSITPA<smiles>c1ccc2c(c1)oc1ncc(-c3ccc(-n4c5ccccc5c5ccccc54)cc3)cc12</smiles>

64 PCz-BFP

图 6 湿法蓝色磷光主体材料

Figure 6 Solution-processed host materials for blue electrophosphorescence 
了有机电致磷光主体材料进展, 小分子电致蓝色磷光器 件中主体材料的进一步发展, 期待解决以下几个主要问 题:

(1)开发性能优良的小分子的双极蓝色磷光主体材 料, 来简化器件结构. 目前在蓝色磷光器件的制备中, 为了实现载流子平衡以提高器件效率, 通常的做法是添 加电子传输材料, 或者在发光层上真空蒸镀空穴阻挡或 电子传输层. 而使用性能优良的双极性主体材料, 不仅 能使载流子在发光层中平衡, 可以简化器件结构, 同时 还可能实现单功能层器件. 这将大大简化器件的制备工 艺, 从而降低生产成本.

(2)设计并合成出新型的适用于湿法的小分子蓝色 磷光主体材料是实现湿法高效率蓝色磷光器件的主要 研究方向之一. 对于掺杂结构的器件, 真空蒸镀通常要 采用双蒸发源或多蒸发源共蒸的方法实现, 材料的掺杂 比例很难控制, 湿法制备时可以通过调控溶液中材料的 浓度, 从而精确控制薄膜中材料的掺杂比例, 这对于制 备多元掺杂的超低掺杂浓度薄膜尤为方便. 可湿法制备 的小分子电致磷光器件虽然在绿色磷光和红色磷光方 面取得了较大的发展. 但可湿法制膜的、适合蓝色磷光 的小分子主体材料偏少, 适用于湿法的具有双极传输特 性的蓝色磷光主体材料更是不多见.

(3)开发性能优良的小分子深蓝色磷光主体材料. 随着 OLED 器件发展到深蓝色系统, 深蓝染料的能隙变 大, 主体材料的能隙也必须跟着变大, 要找到合适的主 体材料变得越来越困难, 并且能隙过大容易造成电子或 空穴注入的问题. 目前小分子深蓝色磷光主体材料偏 少, 适用于湿法的深蓝磷光主体材料更是不多见, 深蓝 色 PhOLED 器件性能需要进一步提高.

\section{References}

[1] Chen, C. H.; Huang, S. W. Organic Electroluminescent Materials \& Devices, Tsinghua University Press, Beijing, 2007 (in Chinese). (陈金金金, 黄孝文, OLED 有机电致发光材料与器件, 清华大学出 版社, 北京, 2007.)

[2] Ma, Y. G.; Zhang, H. Y.; Shen, J. C.; Che, C. M. Synth. Met. 1998, 94, 245.

[3] Baldo, M. A.; O'Brien, D. F.; You, Y.; Shoustikov, A.; Sibley, S.; Thompson, M. E.; Forrest, S. R. Nature 1998, 395, 151.

[4] Kim, D.; Coropceanu, V.; Brédas, J. L. J. Am. Chem. Soc. 2011, 133, 17895 .

[5] Xiao, L. X.; Chen, Z. J.; Qu, B.; Luo, J. X. ; Kong, S.; Gong, Q. H.; Kido, J. Adv. Mater. 2011, 23, 926.

[6] Tao, Y. T.; Yang, C. L.; Qin, J, G. Chem. Soc. Rev. 2011, 40, 2943.

[7] Chaskar, A.; Chen, H. F.; Wong, K. T. Adv. Mater. 2011, 23, 3876.

[8] Wang, C. M.; Fan, Q. L.; Huo, Y. L.; Huang, W. Prog. Chem. 2006, 18,519 (in Chinese). (王传明, 范曲立, 霍岩丽, 黄维, 化学进展, 2006, 18, 519.)

[9] Tao, R.; Qiao, J.; Duan, L.; Qiu, Y. Prog. Chem. 2010, 22, 2255 (in Chinese).

(陶然, 乔娟, 段炼, 邱勇, 化学进展, 2010, 22, 2255.)
[10] Avilov, I.; Marsal, P.; Bredas, J. L.; Beljonne, D. Adv. Mater. 2004, $16,1624$.

[11] Adachi, C.; Kwong, R. C.; Djurovich, P.; Adamovich, V.; Baldo, M. A.; Thompson, M. E.; Forrest, S. R. Appl. Phys. Lett. 2001, 79, 2082.

[12] Holmes, R. J.; Forrest, S. R.; Tung, Y. J.; Kwong, R. C.; Brown, J. J.; Garon, S.; Thompson, M. E. Appl. Phys. Lett. 2003, 82, 2422.

[13] Sasabe, H.; Toyota, N.; Nakanishi, H.; Ishizaka. T.; Pu,Y. J.; Kido, J. Adv. Mater. 2012, 24, 3212.

[14] Tokito, S.; Iijima, T.; Suzuri, Y.; Kita, H.; Tsuzuki, T.; Sato, F. Appl. Phys. Lett. 2003, 83, 569.

[15] Tokito, S.; Iijima, T.; Tsuzuki, T.; Sato, F. Appl. Phys. Lett. 2003, $83,2459$.

[16] He, J.; Liu, H. M.; Dai, Y. F.; Ou, X. M.; Wang, J.; Tao, S. L.; Zhang, X. H.; Wang, P. F.; Ma, D. G. J. Phys. Chem. C 2009, 113, 6761.

[17] Tsai, M. H.; Hong, Y. H.; Chang, C. H.; Su, H. C.; Wu, C. C.; Maroliukstyle, A.; Simokaitiene, J.; Grigalevicius, S.; Grazulevicius, J. V.; Hsu, C. P. Adv. Mater. 2007, 19, 862.

[18] Tsai, M. H.; Ke, T. H.; Lin, H. W.; Wu, C. C.; Chiu, S. F.; Fang, F. C.; Liao, Y. L.; Wong, K. T.; Chen, Y. H.; Wu. C. I. ACS Appl. Mater. Interfaces 2009, 1, 567.

[19] Ding, J. Q.; Zhang, B. H.; Lv, J. H.; Xie, Z. Y.; Wang, L, X.; Jing, X. B.; Wang, F. S. Adv. Mater. 2009, 21, 4983.

[20] Chou, H. H.; Shih, H. H.; Cheng, C. H. J. Mater. Chem. 2010, 20, 798.

[21] Xiao, L. X.; Su, S. J.; Agata, Y.; Lan, H. L.; Kido, J. J. Adv. Mater. 2009, 21, 1271.

[22] Park, M. S.; Choi, D. H.; Lee, B. S.; Lee, J. Y. J. Mater. Chem. 2012, 22, 3099.

[23] Liao, Y. L.; Lin, C. Y.; Liu, Y. H.; Wong, K. T.; Hung, W. Y.; Chen, W. J. Chem. Commun. 2007, 18, 1831.

[24] Li, W.; Qiao, J.; Duan, L.; Wang, L. D.; Qiu, Y. Tetrahedron 2006, 63, 10161.

[25] Shih, P. I.; Chiang, C. L.; Dixit, A. K.;Chen, C. K.; Yuan, M. C.; Lee, R. Y.; Chen, C. T.; Diau, E. W. G.; Shu, C. F. Org. Lett. 2006, $8,2799$.

[26] Shih, P. I.; Chien, C. H.; Wu, F. I.; Shu, C. F. Adv. Funct. Mater. 2007, 17, 3514.

[27] Holmes, R. J.; D'Andrade, B. W.; Forrest, S. R.; Ren, X.; Li, J.; Thompson, M. E. Appl. Phys. Lett. 2003, 83, 3818.

[28] Ren, X. F.; Li, J.; Holmes, R. J.; Djurovich, P. I.; Forrest, S. R.; Thompson, M. E. Chem. Mater. 2004, 16, 4743.

[29] Yeh, S. J.; Wu, M. F.; Chen, C. T.; Song, Y. H.; Chi, Y.; Ho, M. H.; Hsu, S. F.; Chen, C. H. Adv. Mater. 2005, 17, 285.

[30] Tsai, M. H.; Lin, H. W.; Su, H. C.; Ke, T. H.; Wu, C. C.; Fang, F. C.; Liao, Y. L.; Wong, K. T.; Wu, C. I. Adv. Mater. 2006, 18, 1216.

[31] Tsuboi, T.; Liu, S. W.; Wu, M. F.; Chen, C. C. Org. Electon. 2009, $10,1372$.

[32] Hu, D. H.; Lu, P.; Wang, C. L.; Liu, H.; Wang, H.; Wang, Z. M.; Fei, T.; Gu, X.; Ma, Y. G. J. Mater. Chem. 2009, 19, 6143.

[33] Lin, J. J.; Liao, W. S.; Huang, H. J.; Wu, F. I.; Cheng, C. H. $A d v$. Funct. Mater. 2008, 18, 485.

[34] Bin, J. K.; Cho, N. S.; Hong, J. I. Adv. Mater. 2012, 25, 2911.

[35] Brrrows, P. E.; Padmaperuma, A. B.; Sapochak, L. S.; Djurovich, P.; Thompson, M. E. Appl. Phys. Lett. 2006, 88, 183503.

[36] Padmaperuma, A. B.; Sapochak, L. S.; Brrrows, P. E. Chem. Mater. 2006, 18, 2389.

[37] Vecchi, P. A.; Padmaperuma, A. B.; Qiao, H.; Sapochak, L. S.; Brrrows, P. E. Org. Lett. 2006, 8, 4211.

[38] Chen, S. Y.; Xu, X. J.; Liu, Y. Q.; Qiu, W. F.; Yu, G.; Sun, X. B.; Zhang, H. J.; Qi, T.; Lu, K.; Gao, X. K.; Liu, Y.; Zhu, D. B. J. 
Mater. Chem. 2007, 17, 3788.

[39] Fukagawa, H.; Yokoyma, N.; Irisa, S.; Tokito, S. Adv. Mater. 2010, $22,4774$.

[40] Su, S. J.; Sasabe, H.; Pu, Y. J.; Nakayama, K.; Kido, J. Chem. Mater. 2008, 20, 1691.

[41] Rothmann, M. M.; Haneder, S.; Como, E. D.; Lennartz, C.; Schildknecht, C.; Strohriegl, P. Chem. Mater. 2010, 22, 2403.

[42] An, Z. F.; Chen, R. F.; Yin, J.; Xie, G. H.; Shi, H. F.; Tsuboi, T.; Huang, W. Chem. Eur. J. 2011, 17, 10871.

[43] Su, S. J.; Cai, C. H.; Tankmatsu, J.; Kido, J. Org. Electron. 2012, 13, 1937.

[44] Sapochak, L. S.; Cai, X. Y.; Male, J. L.; Burrows, P. E. J. Phys. Chem. C 2008, 112, 7989.

[45] Hsu, F. M.; Chien, C. H.; Shu, C. F.; Lai, C. H.; Hsieh, C. C.; Wang, K. W.; Chou, P. T. Adv. Funct. Mater. 2009, 19, 2834.

[46] Jeon, S. O.; Yook, K. S.; Joo, C. W.; Lee, J. Y. Adv. Funct. Mater. 2009, 19, 3644.

[47] Jeon, S. O.; Yook, K. S.; Joo, C. W.; Lee, J. Y. Adv. Mater. 2010, $22,1872$.

[48] Chou, H. H.; Cheng, C. H. Adv. Mater. 2010, 22, 2468.

[49] Yu, D. H.; Zhao, F. C.; Han, C. M.; Xu, H.; Li, J.; Zhang, Z.; Deng, Z. P.; Ma, D. G.; Yan, P. F. Adv. Mater. 2012, 24, 509.

[50] Yu, D. H., Zhao, F. C.; Zhang, Z.; Han, C. M.; Xu, H.; Li, J.; Ma, D. G.; Yan, P. F. Chem. Commun. 2012, 48, 6157.
[51] Han, C. M.; Zhang, Z. S.; Xu, H.; Li, J.; Xie, G. H.; Chen, R. F.; Zhao, Y.; Huang, W. Angew. Chem., Int. Ed. 2012, 51, 10104.

[52] Liu, H.; Cheng, G.; Hu, D. H.; Shen, F. Z.; Lv ,Y.; Sun, G. N.; Yang, B.; Lu, P.; Ma, Y. G. Adv. Funct. Mater. 2012, 22, 2830.

[53] Arias, A. C.; MacKenzie, J. D.; McCulloch, I.; Rivnay, J.; Salleo, A. Chem. Rev. 2010, 110, 3.

[54] Hou, L. D.; Duan, L.; Qiao, J.; Li, W.; Zhang, D. Q.; Qiu, Y. Appl. Phys. Lett. 2008, 92, 263301.

[55] Ye, S. H.; Liu, Y. Q.; Lu, K.; Wu, W. P.; Du, C. Y.; Liu, Y.; Liu, H. T.; Wu, T.; Yu, G. Adv. Funct. Mater. 2010, 20, 3125.

[56] Ye, S. H.; Chen, J. M.; Lu, K.; Wu, W. P.; Du, C. Y.; Liu, Y.; Wu, T.; Shuai, Z. G.;Yu, G. Adv. Mater. 2010, 22, 4167.

[57] Jiang, W.; Duan, L.; Qiao, J.; Zhang, D. Q.; Dong, G. F.; Wang, L. D.; Qiu, Y. J. Mater. Chem. 2010, 20, 6131.

[58] Jiang, W.; Ge, Z. J.; Cai, P. Y.; Huang, B.; Dai, Y. Q.; Sun, Y. M.; Qiao, J.; Wang, L. D.; Duan, L.; Qiu, Y. J. Mater. Chem. 2012, 22, 12016.

[59] Jiang, W.; Duan, L.; Qiao, J.; Zhang, D. Q.; Dong, G. F.; Wang, L. D.; Qiu, Y. J. Mater. Chem. 2010, 21, 4918.

[60] Jiang, W.; Duan, L.; Qiao, J.; Dong, G. F.; Wang, L. D.; Qiu, Y. Org. Lett. 2011, 13, 3146.

[61] Gong, S. L.; Fu, Q.; Wang, Q.; Yang, C. L.; Zhong, C.; Qin, J. G.; Ma, D. G. Adv. Mater. 2011, 23, 4956.

[62] Lee, C. W.; Lee, J. Y. Adv. Mater. 2013, 25, 596.

(Cheng, F.) 